\title{
ORIGINAL ARTICLE Carfilzomib and the cardiorenal system in myeloma: an endothelial effect?
}

\author{
A Rosenthal, J Luthi, M Belohlavek, KM Kortüm, F Mookadam, A Mayo, R Fonseca, PL Bergsagel, CB Reeder, JR Mikhael and AK Stewart
}

Carfilzomib $(\mathrm{Cfz})$ has been associated with an $\sim 5 \%$ incidence of unexplained and unpredictable cardiovascular toxicity in clinical trials. We therefore implemented a detailed, prospective, clinical cardiac and renal evaluation of $62 \mathrm{Cfz}$-treated myeloma patients, including serial blood pressure (BP), creatinine, troponin, NT-proBNP and pre- and post-treatment echocardiograms, including ejection fraction (EF), average global longitudinal strain and compliance. Pre-treatment elevations in NT-proBNP and BP, as well as abnormal cardiac strain were common. A rise in NT-proBNP occurred frequently post-treatment often without corresponding cardiopulmonary symptoms. A rise in creatinine was common, lessened with hydration and often reversible. All patients had a normal EF pre-treatment. Five patients experienced a significant cardiac event (four decline in EF and one myocardial infarction), of which 2 (3.2\%) were considered probably attributable to Cfz. None were rechallenged with Cfz. The ideal strategy for identifying patients at risk for cardiac events, and parameters by which to monitor for early toxicity have not been established; however, it appears baseline echocardiographic testing is not consistently predictive of toxicity. The toxicities observed suggest an endothelial mechanism and further clinical trials are needed to determine whether or not this represents a class effect or is Cfz specific.

Blood Cancer Journal (2016) 6, e384; doi:10.1038/bcj.2015.112; published online 15 January 2016

\section{INTRODUCTION}

Therapies such as proteasome inhibitors and immunomodulatory drugs have improved overall response rates and survival in multiple myeloma (MM) patients over the last decade. Bortezomib is a first-generation reversible proteasome inhibitor active both as a single agent and in combination. Although infrequent, bortezomib has been associated with cardiotoxicity in animal models ${ }^{1}$ and in clinical experience. ${ }^{2-4}$

Carfilzomib (Cfz) is a next-generation proteasome inhibitor that binds irreversibly, resulting in sustained inhibition of the proteasome with minimal neurotoxicity. ${ }^{5,6} \mathrm{Cfz}$ has shown activity as both a single agent and in combination for patients with relapsed myeloma. ${ }^{7-9}$ Early-phase $1 /$ II studies reported a low but reproducible incidence of cardiorenal toxicity, including hypertension (HTN), pre-renal failure, congestive heart failure and ischemic heart disease. ${ }^{10}$

In a pooled safety analysis from four phase II trials $(n=526)$, Cfz was associated with any grade dyspnea (42\%), HTN (14\%), renal insufficiency (24\%), peripheral edema (24\%) and cardiac events $(22 \%$ ( $7.2 \%$ congestive heart failure) $){ }^{11}$ Cumulatively, $73.6 \%$ of patients experiencing these toxicities had a previous cardiovascular event and $70 \%$ had baseline risk factors for cardiac disease. ${ }^{11}$ Recently, a phase III study of Cfz (K) in combination with lenalidomide and dexamethasone (KRd) showed promising activity with rates of cardiotoxicity comparable to previous reports. ${ }^{12}$

The clinical impact of proteasome inhibitor associated cardiotoxicity has not yet been satisfactorily determined and the pathophysiology is poorly understood. There is no standard approach to cardiac work-up, predicting toxicity or managing events when they occur. In this report, we therefore detail a prospective clinical cardiorenal evaluation of $62 \mathrm{Cfz}$-treated MM patients to gain a better understanding of the impact and to elucidate predictive factors of treatmentrelated cardiorenal events.

\section{METHODS}

Sixty-two patients with MM received Cfz between August 2011 and May 2014 at Mayo Clinic Arizona. Cfz dose, number of cycles and concurrent chemotherapy was recorded. Delivery of hydration pre- and post-treatment was documented. Systolic blood pressure (SBP), creatinine, troponin and NTproBNP were recorded on days 1 and 2 of cycle 1. NT-proBNP was measured on day 8 , whereas creatinine and SBP were collected on day 15. Echocardiograms, with left ventricular ejection fraction (LVEF), average global longitudinal strain and E/e' ratio were performed at baseline, and following four cycles of Cfz. All parameters were measured prospectively; data were collected and analyzed retrospectively with notable cardiorenal events examined for attribution. Institutional Review Board approval for publication of this deidentified clinical data was obtained.

\section{RESULTS}

atient characteristics are presented in Table 1. Sixty-two patients were included for analysis. The median age was 65 years (range 39-78); 60\% were male. Twenty patients were untreated and received $\mathrm{Cfz}$ as part of a clinical trial ( $\mathrm{Cfz}$ with dexamethasone, thalidomide and cyclophosphamide). ${ }^{13}$ Forty-two patients had relapsed disease, with all but one previously treated with bortezomib. Relapsed patients were heavily pre-treated with a median of four prior therapies (range 1-10), including anthracyclines in $21 \%$ and autologous stem cell transplant in $50 \%$. In the relapsed group, 19 patients received $\mathrm{Cfz}$ alone, 10 received $\mathrm{Cfz}$ with cyclophosphamide and 10 received $\mathrm{Cfz}$ with an immunomodulatory agent (lenalidomide or pomalidomide). One patient received $\mathrm{Cfz}$ with pomalidomide and cyclophosphamide, and two patients 


\begin{tabular}{lc}
\hline Table 1. Patient Characteristics & \\
\hline Patient characteristics & $\mathrm{n}=62$ \\
\hline Age & \\
$\quad$ Median & 65 \\
Range & $39-78$ \\
Sex & \\
Male & $60 \%$ \\
Female & $40 \%$ \\
& \\
Disease status & \\
Newly diagnosed & 20 \\
Relapsed & 42 \\
\# Prior therapies (relapsed patients) & \\
$\quad$ Median & \\
Range & 4 \\
Previous treatment & $1-10$ \\
Bortezomib & $n=42$ \\
Stem cell transplant & $41(98 \%)$ \\
Anthracycline & $31(50 \%)$ \\
\end{tabular}

received thalidomide with cyclophosphamide. Dexamethasone was given concurrently in $95 \%$ of patients (4 mg (23/62), $20 \mathrm{mg}(6 / 620)$ and $40 \mathrm{mg}(30 / 62))$.

Patients received Cfz at $20 \mathrm{mg} / \mathrm{m}^{2}$ initially. Doses with subsequent cycles ranged from $27-45 \mathrm{mg} / \mathrm{m}^{2}$ depending on the regimen given on standard dosing days.

Eighteen percentage of patients had baseline HTN (mean $153 \mathrm{mmHg}$; range (146-178 $\mathrm{mmHg}$ )), $90 \%$ of which had been prescribed antihypertensive medication. Systolic HTN was noted in $12 / 41$ patients on day $2(6 / 41$ or $15 \%$, newly $>140)$ and $10 / 41$ on day $15(3 / 41$ or $7 \%$, newly $>140)$. Changes in the opposite direction were also seen, with $11 \%$ of patients having a $>15 \mathrm{mmHg}$ SBP decrease on day 2. Only three patients (two with baseline HTN) had medication adjustments for optimal BP management. Although baseline BP was not generally predictive of future cardiovascular or renal event, it is notable that two hypertensive patients were hospitalized with volume overload during cycle 1.

Hydration $(250-500 \mathrm{ml})$ was delivered to $89 \%$ of patients pre-treatment and $63 \%$ post-treatment. Baseline creatinine was elevated in $33 \%$ of patients on day 1 (mean $1.55 \mathrm{mg} / \mathrm{dl}$; (range $1.3-5.6 \mathrm{mg} / \mathrm{dl})$ ). On day $2,8 / 54$ patients $(15 \%)$ had a rise from baseline in creatinine of $\geqslant 0.3 \mathrm{mg} / \mathrm{dl}$, five of the eight $(63 \%)$ also had elevated baseline creatinine. Patients receiving $<500 \mathrm{ml}$ hydration on day 1 had a significantly higher creatinine on day 2 $(P=0.01)$. Eleven percentage of patients with renal insufficiency experienced improvement in renal function on day 2. Of 26 patients with renal impairment on days 1 or $2,16(61.5 \%)$ had persistently abnormal creatinine on day $8 ; 10$ with a higher creatinine than baseline. Overall, only 3/45 (6\%) patients who had normal creatinine at baseline and received pre-treatment hydration had $a \geqslant$ grade 1 creatinine rise.

Sixteen patients had baseline troponin measurements, all of which were normal $(<0.01 \mathrm{ng} / \mathrm{ml})$ on day 1 . Only two patients, with confounding co-morbidities, subsequently presented with elevated troponins. One patient with cardiac amyloidosis (overlapping with MM) had normal baseline troponin but was hospitalized (cycle 1 day 10) for fluid retention, congestive heart failure and acute kidney injury during which a troponin of $0.029 \mathrm{ng} / \mathrm{ml}$ was recorded. A second patient with a history of coronary artery disease, and no baseline troponin, had an non-ST elevation myocardial infarction (troponin $0.181 \mathrm{ng} / \mathrm{ml}$ ) requiring stent placement on cycle 1 day 2 .

Twenty-two patients had NT-proBNP measured, with a mean baseline of $565 \mathrm{pg} / \mathrm{ml}$ (range $<50-3666 \mathrm{pg} / \mathrm{ml}$ ). After adjusting for age and sex, $82 \%$ had abnormal NT-proBNP levels, with concomitant renal insufficiency in 22\%. Overall, 16/22 (72\%) patients had an increase in NT-proBNP from day 1 to day 2 with an average increase of $1134 \mathrm{pg} / \mathrm{ml}$ above baseline (range $36-6187 \mathrm{pg} / \mathrm{ml}$ ). NT-proBNP continued to rise in $3 / 16$ on day 8 . Post $\mathrm{Cfz}, 52 \%$ of patients had a peak NT-proBNP $\geqslant 500 \mathrm{pg} / \mathrm{ml}$ and $36 \%$ were $\geqslant 1000$ pg/ml (mean 551 pg/ml; ([range 82-9853 pg/ml)); 39\% with abnormal creatinine. Although only 10 patients had a day 8 NT-proBNP, 4 patients had a continual increase $(50 \%$ of which had normal or declining creatinine), 3 had declining values and 3 had no previous measurements for comparison. All but one were above normal (range <50- $28803 \mathrm{pg} / \mathrm{ml}$ ); (Figure 1).

Echocardiographic measurements, including LVEF, strain and E/e' ratio, were assessed at baseline and after four cycles of treatment. All 30 patients ( 19 previously treated (6 with anthracycline exposure); 11 untreated) with baseline echocardiograms had normal LVEF. Three patients (10\%) developed impaired systolic function (LVEF $<50 \%$ ); however, two patients had plausible concurrent alternative explanations: one a documented venous thromboembolism/pulmonary embolism and the other had a low EF detected in the setting of multi-lobar pneumonia and systemic sepsis after cycle 2. The third patient had an unanticipated EF decline during cycle 2 detected preoperatively while hospitalized with a pathologic hip fracture. A fourth patient, with a normal EF following cycle 4, was later found to have a diminished EF during cycle 10 while hospitalized with severe sepsis (Figure 2). All four patients that developed impaired systolic function were pre-treated $(\geqslant 2$ therapies plus stem cell transplant; one prior anthracycline). None continued treatment with $\mathrm{Cfz}$, largely because of progressive disease. In summary, only 1/30 (3\%) patients had an unexplained asymptomatic decrease in $\mathrm{EF}$ detected in context of concurrent hip fracture, although a declining EF was seen concurrently with other major vascular co-morbidity (pulmonary embolism or acute sepsis in three patients).

Baseline cardiac strain (a measure of left ventricular contractility) was abnormal ( $\leqslant-18 \%$ ) in $14 / 25(56 \%)$ patients on pre-treatment echocardiogram. As changes in strain can precede a change in EF, post-treatment strain measurements were also obtained. Strain significantly improved in four patients but also worsened in four. Two patients with worsening strain had a corresponding decline in LVEF but remained within the normal range (68/60\%; 65/58\%). Overall, abnormal cardiac strain was very common before therapy, was not significantly changed during therapy and was not predictive for cardiovascular toxicity.

E/e' ratio was used as a surrogate marker for the left ventricular end diastolic pressure and served as a measure of ventricular compliance. By using a ratio of $<15$ as normal, $46 \%$ of the 28 patients with an $\mathrm{E} / \mathrm{e}^{\prime}$ ratio had abnormal ratios before $\mathrm{Cfz}$

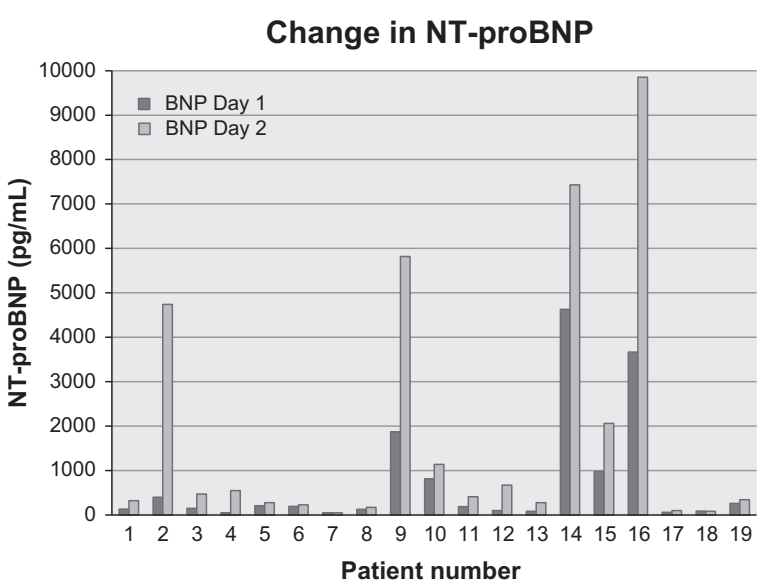

Figure 1. Change in NT-proBNP following treatment with carfilzomib (days 1 and 2). 
treatment. Post-treatment, an equal number of patients, demonstrated worsening or improvement of this parameter.

\section{DISCUSSION}

In this detailed prospective analysis of cardiorenal toxicity following $\mathrm{Cfz}$, significant elevations in SBP and NT-proBNP were common following treatment. Cardiac peptides were frequently elevated at baseline (59\%); however, abnormal values are difficult to interpret as they are subject to change based on age, sex, body mass index and renal function. ${ }^{14,15} \mathrm{NT}$-proBNP rose significantly in $84 \%$ of patients immediately following administration of $\mathrm{Cfz}$ even in the absence of clinically relevant cardiopulmonary symptoms. Creatinine elevation post-Cfz was common amongst those with baseline renal insufficiency, whereas hydration pre-treatment appeared renal protective in those with normal creatinine at baseline.

Baseline echocardiographic measurements, including LVEF, strain and E/e' ratio, were not consistently predictive of subsequent cardiotoxicity. Five patients with relapsed/refractory MM had significant cardiac events (Table 2). As described above, four had a decline in LVEF post-treatment, whereas one patient experienced an non-ST elevation myocardial infarction during cycle 1. In only one patient was the decline in EF an isolated event (3\%). In all of these patients no further $\mathrm{Cfz}$ was administered and follow-up echocardiograms are not available making it difficult to draw any conclusions about recovery of cardiac function, although there is anecdotal data to suggest reversibility. ${ }^{16}$ Our experience correlates with published data of cardiac toxicity and indicates that baseline testing of BNP and cardiac function by echocardiogram is not helpful. ${ }^{2}$ Notably, two of the four cardiac events documented were in patients with amyloid and ischemic heart disease, suggesting that this drug should be used with caution in

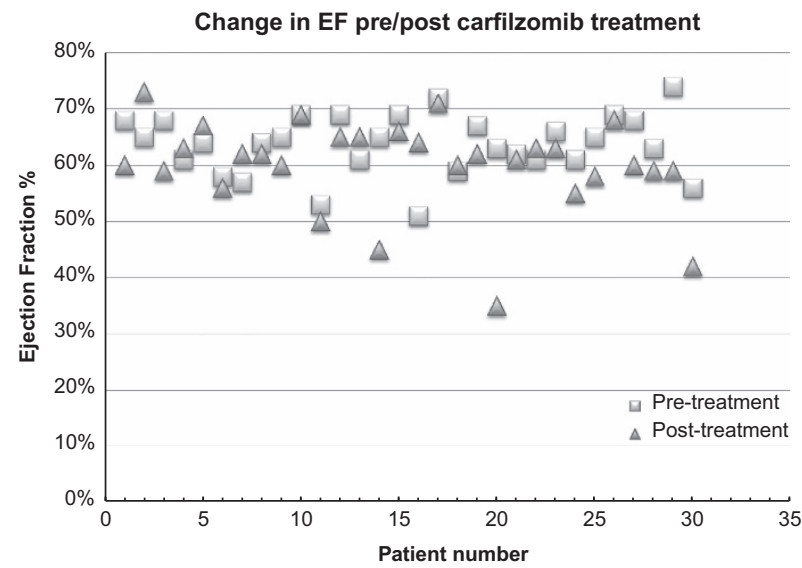

Figure 2. Change in ejection fraction pre-/post-treatment with carfilzomib (*post-treatment refers to ejection fraction assessment following four cycles of treatment with carfizomib).

\begin{tabular}{|c|c|c|c|}
\hline Patient & $\begin{array}{l}\text { Pre-/post- } \\
\text { treatment LVEF }\end{array}$ & $\begin{array}{l}\text { Clinical } \\
\text { scenario }\end{array}$ & $\begin{array}{l}\text { Attributable } \\
\text { to } \mathrm{Cfz} \text { ? }\end{array}$ \\
\hline 1 & $65 / 45 \%$ & $\begin{array}{l}\text { Multilobar } \\
\text { pneumonia }\end{array}$ & Unlikely \\
\hline 2 & $61 / 45 \% ; 40 \%$ cycle 10 & Systemic sepsis & Unlikely \\
\hline 3 & $63 / 35 \%$ & VTE/PE & No \\
\hline 4 & $56 / 42 \%$ & Hip fracture (cycle 2) & Probable \\
\hline 5 & None/54\% & $\mathrm{MI} /$ known CAD & Probable \\
\hline
\end{tabular}

the presence of baseline cardiac disease and possibly cardiac amyloidosis.

Relapsed/refractory patients are more likely to have co-morbidities. In our experience, significant cardiac events were only observed in previously treated patients. This finding concurs with significantly higher degrees of toxicity in the FOCUS late stage myeloma clinical trial when compared with ASPIRE and ENDEAVOR. ${ }^{12,17,18}$

Cardiovascular toxicity, although infrequent, appears to occur early in the course of treatment. Several studies have now demonstrated safety with long-term administration of $\mathrm{Cfz}{ }^{10,12,19}$ The long-term tolerability and increasing evidence that cumulative toxicity is uncommon, make $\mathrm{Cfz}$ an appealing option for consolidation or maintenance strategies aimed at deepening and prolonging response. $^{20}$

In summary, the cardiorenal impact of $\mathrm{Cfz}$ is relatively infrequent yet more common in heavily pre-treated patients and those with baseline cardiorenal dysfunction. The toxicity appears to have an endothelial component-HTN, reversible rise in creatinine (partially preventable by hydration), common acute rise in NT-proBNP and lack of evidence for isolated structural cardiomyopathy. Whether these observations represent a class effect or are unique to $\mathrm{Cfz}$ alone is unknown. Prospective controlled studies with longer term follow-up and bortezomib-treated controls are ongoing (ENDEAVOR $^{18}$ (NCT01568866) and CLARION (NCT01818752)).

\section{CONFLICT OF INTEREST}

KAS is a consultant for Celgene, Novartis, BMS and AMGEN. LB is a consultant for Onyx. RF is a consultant for Medtronic, Otsuka, Celgene, Genzyme, BMS, Lilly, Onyx Binding Site, Millenium and AMGEN, and has received research funding from Cylene and Onyx. JM has received research funding from Celgene, Abbvie, Onyx, and Sanofi. The remaining authors declare no conflict of interest.

\section{REFERENCES}

1 Nowis D, Mączewski M, Mackiewicz U, Kujawa M, Ratajska A, Wieckowski MR et al. Cardiotoxicity of the anticancer therapeutic agent bortezomib. Am J Pathol 2010; 176: $2658-2668$.

2 Richardson PG, Sonneveld P, Schuster MW, Irwin D, Stadtmauer EA, Facon T et al. Bortezomib or high-dose dexamethasone for relapsed multiple myeloma. N Eng J Med 2005; 352: 2487-2498.

3 Honton B, Despas F, Dumonteil N, Rouvellat C, Roussel M, Carrie D et al. Bortezomib and heart failure: case-report and review of the French Pharmacovigilance database. Fundam Clin Pharmacol 2014; 28: 349-352.

4 Subedi A, Sharma L, Shah B. Bortezomib-induced acute congestive heart failure: a case report and review of literature. Ann Hematol 2014; 93: 1797-1799.

5 Demo S, Kirk C, Aujay M, Buchholz T, Dajee M, Ho M et al. Antitumor activity of PR-171, a novel irreversible inhibitor of the proteasome. Cancer Res 2007; 67: 6383-6391.

6 Arastu-Kapur S, Anderl J, Kraus M, Parlati F, Shenk K, Lee S et al. Nonproteasomal targets of the proteasome inhibitors bortezomib and carfilzomib: a link to clinical adverse events. Clin Cancer Res 2011; 17: 2734-2743.

7 Vij R, Siegel DS, Jagannath S, Jakubowiak AJ, Stewart AK, McDonagh K et al. An open-label, single-arm, phase 2 study of single-agent carfilzomib in patients with relapsed and/or refractory multiple myeloma who have been previously treated with bortezomib. Br J Haematol 2012; 158: 739-748.

8 Kortuem KM, Stewart AK. Carfilzomib. Blood 2013; 121: 893-897.

9 Jagannath S, Vij R, Stewart AK, Trudel S, Jakubowiak AJ, Reiman $T$ et al. An open-label single-arm pilot phase II study (PX-171-003-A0) of low-dose, singleagent carfilzomib in patients with relapsed and refractory multiple myeloma. Clin Lymphoma Myeloma Leuk 2012; 12: 310-318.

10 Siegel D, Martin T, Wang M, Vij R, Jakubowiak A, Lonial S et al. A phase 2 study of single-agent carfilzomib (PX-171-003-A1) in patients with relapsed and refractory multiple myeloma. Blood 2012; 120: 2817-2825.

11 Siegel D, Martin T, Nooka A, Harvey RD, Vij R, Niesvizky R et al. Integrated safety profile of single-agent carfilzomib: experience from 526 patients enrolled in 4 phase II clinical studies. Haematologica 2013; 98: 1753-1761.

12 Stewart AK, Rajkumar SV, Dimopoulos MA, Masszi T, Špička I, Oriol A et al. Carfilzomib, lenalidomide, and dexamethasone for relapsed multiple myeloma. N Eng J Med 2015; 372: 142-152.

13 Mikhael JR, Reeder CB, Libby EN, Costa LJ, Bergsagel PL, Buadi F et al. Phase lb/I trial of CYKLONE (cyclophosphamide, carfilzomib, thalidomide and dexamethasone) for newly diagnosed myeloma. Br J Haematol 2015; 169: 219-227. 
14 Raymond I, Groenning BA, Hildebrandt PR, Nilsson JC, Baumann M, Trawinski J et al. The influence of age, sex and other variables on the plasma level of $\mathrm{N}$-terminal pro brain natriuretic peptide in a large sample of the general population. Heart 2003; 89: 745-751.

15 Anwaruddin S, Lloyd-Jones DM, Baggish A, Chen A, Krauser D, Tung R et al. Renal function, congestive heart failure, and amino-terminal pro-brain natriuretic peptide measurement: results from the ProBNP Investigation of Dyspnea in the Emergency Department (PRIDE) Study. J Am Coll Cardiol 2006; 47: 91-97.

16 Grandin EW, Ky B, Cornell RF, Carver J, Lenihan DJ. Patterns of cardiac toxicity associated with irreversible proteasome inhibition in the treatment of multiple myeloma. J Card Fail 2015; 21: 138-144.

17 Ludwig H, Masszi T, Pertucci MT, Palumbo A, Rosinol L, Nagler A et al. Carfilzomib vs low-dose corticosteroids and optional cyclophosphamide in patients with relapsed and refractory multiple myeloma: A phase 3 study (FOCUS). Ann Oncol 2014; 25.

18 Dimopoulos MA, Moreau P, Palumbo A, Joshua DE, Pour L, Hajek R et al. Carfilzomib and dexamethasone (Kd) vs bortezomib and dexamethasone (Vd) in patients (pts) with relapsed multiple myeloma (RMM): Results from the phase III study ENDEAVOR. J Clin Oncol (ASCO Meeting Abstracts) 2015; 33: 8509.
19 Jagannath S, Vij R, Kaufman JL, Martin T, Niesvizky R, Gabrail NY et al. Long-term treatment and tolerability of the novel proteasome inhibitor carfilzomib (cfz) in patients with relapsed and/or refractory multiple myeloma (R/R MM). Blood (ASH Annual Meeting Abstracts) 2010; 116: 1953.

20 Ludwig H, Durie BGM, McCarthy P, Palumbo A, San Miguel J, Barlogie B et al. IMWG consensus on maintenance therapy in multiple myeloma. Blood 2012; 119: 3003-3015.

\section{(c) (i)}

This work is licensed under a Creative Commons Attribution 4.0 International License. The images or other third party material in this article are included in the article's Creative Commons license, unless indicated otherwise in the credit line; if the material is not included under the Creative Commons license, users will need to obtain permission from the license holder to reproduce the material. To view a copy of this license, visit http://creativecommons.org/licenses/ by/4.0/ 